infections. There was no leukemic transformation or deaths in our study group.

Conclusion Timely and early referral to specialist services will not only prevent morbidity, enable appropriate follow up with early recognition of complications and institution of preventive patient care but also promote family's understanding of and coping with the syndrome.

\section{PS-086 QUESTIONNAIRE STUDY FOR ASSESSMENT OF QUALITY OF LIFE (QOL) IN CHILDREN WITH SHWACHMAN DIAMOND SYNDROME (SDS)}

${ }^{1}$ A Silwal, ${ }^{2}$ SE Kinsey, ${ }^{3} \mathrm{~J}$ Puntis. 'Department of Paediatrics, Leeds General Infirmary, Leeds, UK: ${ }^{2}$ Department of Paediatric Haematology, Leeds General Infirmary, Leeds, UK; ${ }^{3}$ Department of Paediatric Gastroenterology, Leeds General Infirmary, Leeds, UK

10.1136/archdischild-2014-307384.381

SDS is a multisystem disorder with heterogenous clinical presentation. Neuro-cognitive impairments, intellectual disability and behavioural difficulties have been reported in previous studies.

Methods and patients We used validated child and parent reporting questionnaires (CHQ-CF87, CHQ-PF50) to explore QoL in children with SDS aged 5-18 years (followed at SDS multidisciplinary clinic at Leeds). 7 children and 1 of their parent completed the self-administered questionnaires. Data was analysed and interpreted as per scoring and interpretation manual provided with the questionnaires. Ethics approval was obtained.

Results Parents scored their child below reference sample for global health and behaviour; they recognised that they worried about their child's health more than other people. There was discrepancy in scoring on some health domains between child and parent CHQ which could represent child's lack of insight or better scoring by parents comparing their child's health and behaviour with their peers.

Qualitative analysis revealed that majority of children reported good health and all experienced mild pain or discomfort. Most were happy, got on with friends, but admitted to arguing and recognised their health/behaviour caused family tension.

Parents reported difficulty concentrating, paying attention and argumentative behaviour. They expressed uncertainty about their child having a healthy life and reported their health/behaviour caused disruption of everyday family activities. Some parents reported their children having difficulties in activities like eating, dressing or going to toilet alone.

Conclusions Our findings identify important qualitative areas of concern and highlight potential areas for providing targeted support to children with SDS and their families.

\section{PS-087 WITHDRAWN}

\section{PS-088 TREATMENT OF PRIMARY IMMUNE THROMBOCYTOPENIA BY PREDNISOLONE: 4 MG/KG/J FOR 4 DAYS VERSUS 2 MG/KG/J FOR 6-8 WEEKS}

'L Sfaihi, 'S Alibi, ${ }^{2}$ Djedidi, 'S Ben Ameur, ${ }^{2}$ Damak, ${ }^{1} \mathrm{M}$ Hachicha. ${ }^{1}$ Pediatrics, Hédi Chaker University Hospital, Sfax, Tunisia; ${ }^{2}$ Community Medicine and Epidemiology, Hédi Chaker University Hospital, Sfax, Tunisia
Background and aims Primary immune thrombocytopenia (ITP) is characterised by accelerated platelet destruction. Corticosteroids have been shown to increase platelet counts. The objective of our study was to compare the treatment with prednisolone at a dose of $4 \mathrm{mg} / \mathrm{kg} /$ day for 4 days (Protocol P1) to the treatment with prednisolone at the dose of $2 \mathrm{mg} / \mathrm{kg} /$ day for 6-8 weeks (Protocol P2).

Methods We conducted a comparative analytical study between 2 groups: first group (G1) formed by ITP cases followed prospectively between 2010 and 2013. These patients received protocol P1. The second group (G2) collecting the same number of patients followed retrospectively between 1999 and 2008. These patients received protocol $\mathrm{P} 2$.

Results Our study included 24 cases: 12 cases in G1 and 12 cases in G2. The response on day three was $25 \%$ for G1 and $0 \%$ for G2. The response on the fifth day was $66.7 \%(\mathrm{G} 1)$ and $54.5 \%$ (G2). The median time to response was 5 days for the 2 groups, with extremes ranging from 3 to 30 days (G1) and 5 to 30 days (G2) (p: 0.09). The complete response occurred in 15 days with a range of 5-90 days for G1 and 30 days with a range of 5-90 days for G2 ( $\mathrm{p}=0.04)$.

Conclusion Within the limits of our study, we are able to show the interest of high dose of prednisolone during 4 days in the treatment of acute ITP.

\section{PS-089 WHICH DECISION RULES MEET METHODOLOGICAL STANDARDS IN CHILDREN WITH FEBRILE NEUTROPENIA? RESULTS OF A SYSTEMATIC REVIEW AND ANALYSIS}

${ }^{1} \mathrm{M}$ Delebarre, ${ }^{1} \mathrm{E}$ Macher, ${ }^{2} \mathrm{~F}$ Mazingue, ${ }^{3} \mathrm{~A}$ Martinot, ${ }^{3} \mathrm{~F}$ Dubos. ${ }^{1}$ Pediatric Hematology Unit Jeanne de Flandre Hospital Pediatric Emergency Unit and Infectious Diseases R. Salengro Hospital, CHRU Lille and University Lille Nord-de-France UDSL, Lille, France; ${ }^{2}$ Pediatric Hematology Unit Jeanne de Flandre Hospital, CHRU Lille and University Lille Nord-deFrance UDSL, Lille, France; ${ }^{3}$ Pediatric Emergency Unit and Infectious Diseases $R$. Salengro Hosp., CHRU Lille and University Lille Nord-de-France UDSL, Lille, France

\subsection{6/archdischild-2014-307384.383}

Background and aim Clinical decision rules (CDRs) have sought to identify the few children with chemotherapy-induced febrile neutropenia $(\mathrm{FN})$ really at risk of severe infection to reduce the invasive procedures and costs for those at low risk. Several reports have shown that most rules do not perform well enough to be clinically useful. Our objective was to analyse the derivation methods and validation procedures of these CDRs.

Methods A systematic review using Medline, Ovid, Refdoc, and the Cochrane Library through December 2012 searched for all CDRs predicting the risk of severe infection and/or complications in children with chemotherapy-induced FN. Their methodological quality was analysed by 17 criteria for deriving and validating a CDR identified in the literature. The criteria published by the Evidence Based Medicine Working Group were applied to the published validations of each CDR to assess their level of evidence.

Results The systematic research identified 612 articles and retained 12 that derived CDRs. Overall the CDRs met a median of $65 \%$ of the methodological criteria. The criteria met least often were that the rule made clinical sense, or described the course of action, or that the variables and the CDR were reproducible. Only one CDR, developed in South America, met all methodological criteria and provided the highest level of evidence; unfortunately it was not reproducible in Europe. 